\title{
Embolization with the Amplatzer Vascular Plug in TIPS Patients
}

\author{
Peter M. T. Pattynama · Alexandra Wils • \\ Edwin van der Linden · Lukas C. van Dijk
}

Received: 21 March 2007/Revised: 21 March 2007/ Accepted: 20 April 2007 / Published online: 2 June 2007

(C) Springer Science+Business Media, LLC 2007

\begin{abstract}
Vessel embolization can be a valuable adjunct procedure in transjugular intrahepatic portosystemic shunt (TIPS). During the creation of a TIPS, embolization of portal vein collaterals supplying esophageal varices may lower the risk of secondary rebleeding. And after creation of a TIPS, closure of the TIPS itself may be indicated if the resulting hepatic encephalopathy severely impairs mental functioning. The Amplatzer Vascular Plug (AVP; AGA Medical, Golden Valley, MN) is well suited for embolization of large-diameter vessels and has been employed in a variety of vascular lesions including congenital arteriovenous shunts. Here we describe the use of the AVP in the context of TIPS to embolize portal vein collaterals $(n=8)$ or to occlude the TIPS $(n=2)$.
\end{abstract}

Keywords Interventional radiology - Embolization ·

Amplatzer Vascular Plug · Varices · Transjugular intrahepatic portosystemic shunt

Portal vein collaterals supplying esophageal varices may give high risk for secondary rebleeding. Those collaterals might require embolization to decrease this risk, despite transjugular intrahepatic portosystemic shunt (TIPS) placement. Embolization of the collaterals may be particularly difficult when these vessels are large. Moreover,

P. M. T. Pattynama $\cdot$ A. Wils · E. van der Linden ·

L. C. van Dijk

Department of Radiology, Erasmus University Medical Center,

's-Gravendijkwal 230, 3015 CE Rotterdam, The Netherlands

P. M. T. Pattynama ( $₫)$

Department of Radiology, Suite Hs-210, Erasmus University

Medical Center, P.O. Box 2040, 3000 CA Rotterdam,

The Netherlands

e-mail: p.m.t.pattynama@erasmusmc.nl after creation of a TIPS, closure of the TIPS itself may be indicated when hepatic encephalopathy severely impairs mental functioning. The Amplatzer Vascular Plug (AVP; AGA Medical, Golden Valley, MN) is a device well suited to embolize large vessels and has been employed in a variety of vascular lesions such as pulmonary arteriovenous malformations and congenital shunts [1,2].

This report describes the use of the AVP in the context of TIPS to embolize portal vein collaterals $(n=8)$ or to occlude the TIPS $(n=2)$.

\section{Description of Procedures}

\section{Embolization of Portal Vein Collaterals}

Eight patients were referred for TIPS, seven because of multiple recurrent bleeding episodes from esophageal varices $(n=5)$, gastric fundus varices $(n=1)$, or both $(n=1)$ that could not be managed by endoscopic therapy. One other patient $(n=1)$ was in hypovolemic shock because of recurrent massive bleeding from rectal hemorrhoids. Three TIPS procedures were elective and five were done as emergency procedures for uncontrolled bleeding after initial hemodynamic stabilization had been achieved using blood transfusions, endoscopic treatment, intravenous octreotide infusion, and/or placement of a Sengstaken double balloon tube, alone or in combination. TIPS procedures were carried out under general anesthesia.

As an adjunct procedure, we obliterated the portal vein collaterals supplying the bleeding varices using AVP embolization (Figs. 1 and 2). This was done either before or after creation of the TIPS based on operator preference and clinical circumstances. Embolization early in the procedure with early control of the potential bleeding site was 
considered relevant in emergency procedures in hemodynamically unstable patients. Also, at least theoretically, AVP embolization may be done more easily prior to TIPS whenever the collateral target vein is still widened because of portal hypertension and easily opacified because of preferential flow. After the creation of a TIPS both these phenomena may have been altered.

Whenever AVP embolization preceded TIPS placement, the guiding catheter for AVP delivery was introduced transhepatically into the right portal vein. At our institution the portal vein is routinely catheterized prior to TIPS with a 3 - or 4-Fr catheter to visualize the portal vein as a fluoroscopic target for transjugular transhepatic puncture. For

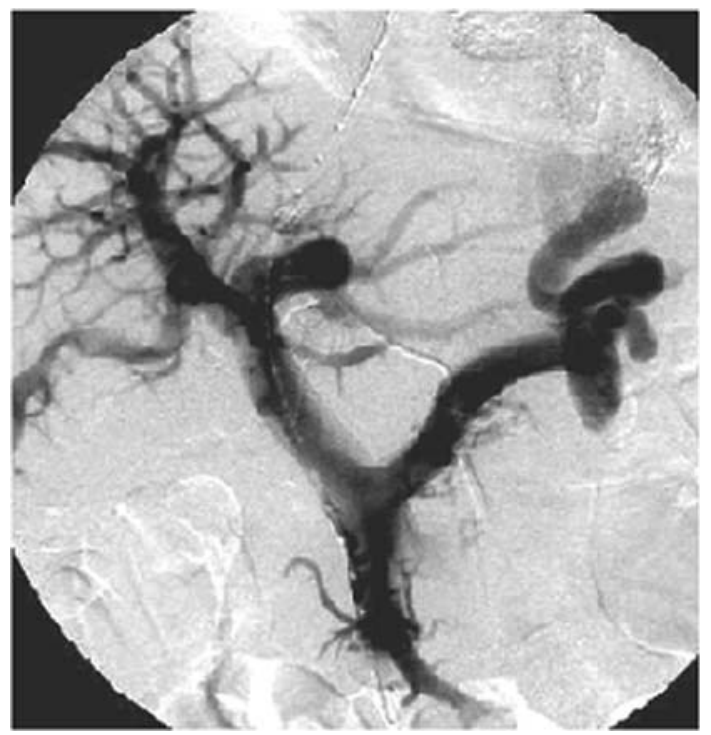

Fig. 1 Portal venous angiogram in a 53-year-old patient referred for TIPS creation because of recurrent variceal bleeding not controlled by medication plus multiple treatments with endoscopic band ligation and sclerotherapy. The varices are supplied by a single coronary vein transhepatic AVP placement this catheter was exchanged for a 6- to 8-Fr hepatic introducer sheath. After AVP embolization and initial creation of the hepatic-to-portal vein shunt tract for TIPS, the hepatic introducer sheath was withdrawn in multiple small successive steps over the course of the remainder of the procedure, so that a solid sealing clot could form in the hepatic parenchymal tract. No additional embolization of the tract was performed. Whenever vein embolization was performed after the creation of a TIPS, AVP placement was done via the transjugular route.

In all patients, the large-diameter coronary/gastric vein supplying extensive esophageal/gastric varices was embolized using a single AVP plug 10,12 , or $14 \mathrm{~mm}$ in diameter. For placement, a 6- to 8-Fr guiding catheter was first negotiated into the proximal part of the coronary vein. Then the AVP device was advanced through the guiding catheter and initially deployed by retracting the guiding catheter but without detaching the device. A control injection was made, and if needed, the AVP was retrieved into the guiding catheter and repositioned. When in a satisfactory position, the AVP was detached by anticlockwise rotation of the AVP guide wire to unscrew it from the AVP device proper. Within minutes of deployment, the collateral flow through the coronary vein had completely stopped. TIPS creation was done by placement of e-PTFEcovered stent-grafts (Viatorr; Gore, Flagstaff, AZ) 8-10 $\mathrm{mm}$ in diameter and $6 \pm 2 \mathrm{~cm}$ long, resulting in a postprocedural portosystemic pressure difference of 5-10 mm $\mathrm{Hg}$. All patients were hemodynamically stable immediately after the procedure.

During follow-up there were no recorded episodes of rebleeding with a follow-up of 1 year maximum. The patient who was treated for bleeding rectal hemorrhoids died after 3 days because of combined liver and respiratory failure. Another patient who received emergency TIPS
Fig. 2 The same patient as in Fig. 1 immediately following TIPS creation and AVP embolization of the coronary vein (a; thin arrow). The unsubtracted image (b) shows the radiopaque sclerosing agent from prior endoscopic sclerotherapy (fat arrow) in the esophageal varices
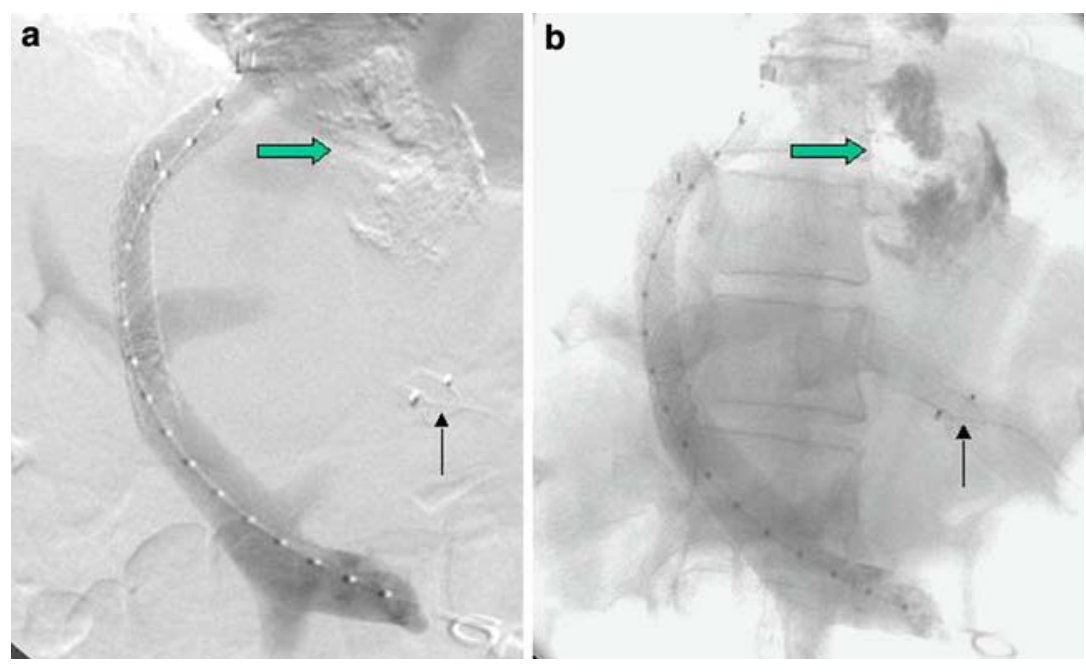
while in hypovolemic shock due to massive unstoppable bleeding from esophageal varices died 46 days after the procedure because of liver and kidney failure complicated by ileus.

\section{TIPS Occlusion}

A 47-year-old male was referred for closure of a TIPS that had been created 3 years earlier for alcoholic Child A liver cirrhosis with recurrent episodes of esophagus variceal bleeding. He now presented with problems of amnesia, difficulties concentrating, and a general, overwhelming feeling of tiredness. Spectral EEG findings were consistent with grade 2 hepatic encephalopathy. The symptoms had not improved using conservative treatment. At angiography, a patent TIPS was seen, with a portosystemic pressure difference of $10 \mathrm{~mm} \mathrm{Hg}$. Closure of the TIPS with a 14-mm AVP device (Fig. 3) resulted in a dramatic improvement in cognitive functioning and normalization of the spectral EEG. Two years after closure of the TIPS, the patient is still free of variceal bleeding. Recently, however, he developed esophageal varices grades I-II, which are currently treated with band ligation. This will be repeated every 2 weeks until complete obliteration.

Another patient, a 65-year-old male, had been referred for TIPS placement for refractory ascites following liver transplantation, which required weekly paracentesis. In the days following placement of a 10-mm-diameter e-PTFEcovered stent-graft TIPS, liver failure developed, necessitating its closure. Closure of the TIPS was technically successfully achieved using a 16-mm-diameter AVP but did not substantially improve the clinical status. Because of liver failure and refractory ascites, the patient underwent a second liver transplantation 2 days after TIPS occlusion.

\section{Discussion}

In this report we have described the use of AVP embolization in the context of TIPS. For closure of collateral veins supplying bleeding varices, we consider the AVP a useful device in a defined subset of patients. The AVP's relative advantages are its efficiency to occlude largediameter vessels in a single-step procedure and its reliability of placement. Unlike standard embolization coils, the AVP can be positioned precisely, its position can be checked with contrast injections before actual detachment, and it can be repositioned if required. Migration of the AVP during or after deployment is unlikely. Disadvantages of the AVP include the higher cost of the device itself and of the additionally required guiding catheter. Furthermore, the 6- to 8-Fr guiding catheter markedly increases the bulk of the introducer system relative to the 4- to 5 -Fr catheter alternatively used for coil embolization. In our practice we are inclined to use the AVP whenever there is a single, large-diameter collateral vein which would otherwise require the insertions of multiple embolization coils and/or whenever accurate placement of the embolic device close to the portal vein may be critical.

Embolization of portal vein collaterals to esophageal varices may be indicated even after successful portal venous decompression by creation of a TIPS. Especially in the life-threatening emergency situation of acute esophageal bleeding in hemodynamically unstable patients, we feel more confident providing additional control of the bleeding site by hemodynamically separating the weakwalled varices from the high-flow and potentially highpressure portal venous bed. TIPS has proven efficient in the secondary prevention of variceal bleeding but this protection is not absolute. The rebleeding rate of TIPS alone

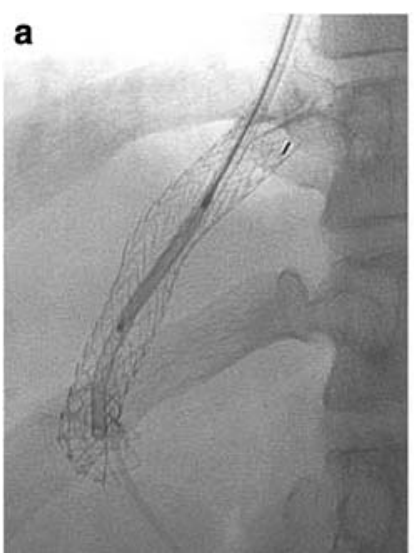

Fig. 3 AVP device for TIPS occlusion in a patient with lifeincapacitating hepatic encephalopathy. The 14-mm-diameter AVP is positioned through a guiding catheter in the central portion of the 10mm-diameter e-PTFE stent-graft (a). By retraction of the guide wire,
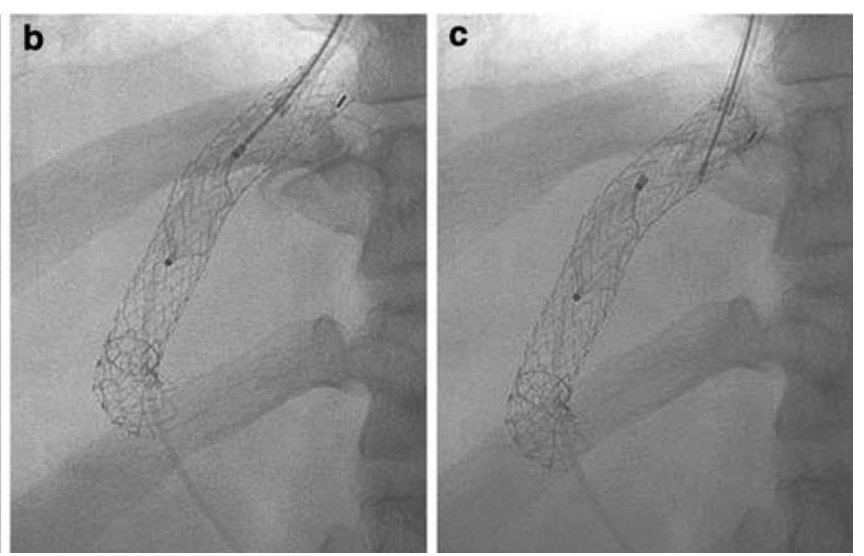

the unconstrained AVP sets in the intended place (b). At this point, the AVP can still be retracted into the guiding catheter if in an unsatisfactory position. The AVP is fully deployed by unscrewing the guide wire from the AVP device proper (c) 
(without embolization) has been reported as between $9 \%$ and $40.6 \%$, with a median of $18.4 \%$ [3]. These values apply to bare-stented TIPS and initial experience suggests that the rebleeding rate is lower with e-PTFE-covered stented TIPS [4]. Whether or not performing embolization of the portal vein collaterals as an adjunct procedure provides additional protection against rebleeding is unknown. Under the assumption that it does, we routinely combine TIPS with embolization of all venous collaterals supplying the esophageal varices when creating a TIPS in the context of variceal bleeding.

Another potential use for the AVP device is for closure of the TIPS itself, in circumstances where hepatic encephalopathy and/or liver failure because of the shunt may prove to be life-incapacitating. Here, the effectiveness of the AVP in occluding large-diameter high-flow vessels, its resistance to migration, and its ease of placement are particularly helpful.

A potential concern when occluding a TIPS is the acute increase in portal venous pressure, which can lead to recurrence of variceal bleeding and ascites and, also, to hemodynamic changes with increased cardiac loading [5].

As mentioned, we routinely place a transhepatic catheter into the portal vein as a target for puncturing the portal vein during TIPS creation. In our experience, this increases the control over the exact entrance point into the portal vein and also reduces the number of puncture attempts while creating the TIPS tract. We have found that the reduced procedure time for the TIPS procedure proper more than makes up for the extra time it takes to insert this initial transhepatic catheter into the portal vein. We have encountered no complications because of this approach in more than 100 TIPS procedures.
To our knowledge, this is the second reported use of the AVP in the portal venous system. In a recent case report, Kessler and Trerotola described a patient with gastric varices and TIPS in whom they used the AVP device for transhepatic obliteration of the portal vein collateral supplying the varices [6], a patient similar to one of our patients with flow-mediated gastric varices. We would like to emphasize that the AVP may be considered also for embolization of the more common collateral veins to esophageal varices and, if need be, the TIPS stent itself.

\section{References}

1. Rossi M, Rebonato A, Greco L, et al. (2006) A new device for vascular embolization: report on case of two pulmonary arteriovenous fistulas embolization using the Amplatzer Vascular Plug. Cardiovasc Intervent Radiol 29:902-906

2. Ferro C, Rossi UG, Bovio G, et al. (2007) Percutaneous transcatheter embolization of a large pulmonary arteriovenous fistula with an Amplatzer Vascular Plug. Cardiovasc Intervent Radiol 30:328-331

3. Burroughs AK, Vangeli M (2002) Transjugular intrahepatic portosystemic shunt versus endoscopic therapy: randomized controlled trials for secondary prophylaxis of variceal bleeding: an updated meta-analysis. Scand J Gastroenterol 37:249-252

4. Bureau C, Garcia-Pagan JC, Otal P, et al. (2004) Improved clinical outcome using polytetrafluoroethylene-coated stents for TIPS: results of a randomized study. Gastroenterology 126:469-475

5. Paz-Fumagalli R, Crain MR, Mewissen MW, et al. (1994) Fatal hemodynamic consequences of therapeutic closure of a transjugular intrahepatic portosystemic shunt. J Vasc Interv Radiol 5(6):831-834

6. Kessler J, Trerotola SO (2006) Use of the Amplatzer Vascular Plug for embolization of a large retroperitoneal shunt during transjugular intrahepatic portosystemic shunt creation for gastric variceal bleeding. J Vasc Interv Radiol 17:135-140 